\title{
Comparison Between Neutrophil-Lymphocyte Ratio And Systemic Immune-Inflammation Index As Predictors Of One-Year Survival In Patients With Untreated Advanced Hepatocellular Carcinoma
}

\section{Irsan Hasan}

RSUPN Dr Cipto Mangunkusumo: Rumah Sakit Dr Cipto Mangunkusumo Lutfie Lutfie ( $D$ lutfie040991@yahoo.com)

RSUPN Dr Cipto Mangunkusumo: Rumah Sakit Dr Cipto Mangunkusumo Ikhwan Rinaldi

RSUPN Dr Cipto Mangunkusumo: Rumah Sakit Dr Cipto Mangunkusumo Juferdy Kurniawan

RSUPN Dr Cipto Mangunkusumo: Rumah Sakit Dr Cipto Mangunkusumo Imelda Maria Loho

Dharmais Cancer Hospital: Rumah Sakit Dharmais Pusat Kanker Nasional

\section{Research Article}

Keywords: Hepatocellular carcinoma, neutrophil-lymphocyte ratio, systemic immune-inflammation index, one-year survival

Posted Date: October 21st, 2021

DOI: https://doi.org/10.21203/rs.3.rs-982508/v1

License: (c) (i) This work is licensed under a Creative Commons Attribution 4.0 International License. Read Full License

Version of Record: A version of this preprint was published at Journal of Gastrointestinal Cancer on January 31st, 2022. See the published version at https://doi.org/10.1007/s12029-021-00796-7. 


\section{Abstract \\ Background}

Patients with hepatocellular carcinoma (HCC) generally only come for treatment when cancer has reached an advanced stage, with very limited treatment options. There has not been an accurate predictor marker to be able to identify which group of patients may have better survival. This study wanted to analyze the role of the inflammatory status indices as predictors of one-year survival in patients with advanced HCC who did not undergo therapy.

\section{Methods}

This study has a retrospective cohort design using secondary data on subjects with advanced HCC who did not undergo therapy at Cipto Mangunkusumo Hospital and Dharmais Hospital. The Neutrophillymphocyte ratio (NLR) and Systemic immune-inflammation index (SII) were evaluated for their role as predictors of one-year survival based on the Area Under Receiving Operator Curve (AUROC). The best optimal cutoff for NLR and SII was decided based on the Youden index, followed by survival analysis based on those cutoffs. Confounding factors were analyzed with multivariate cox regression analysis.

\section{Results}

A total of 196 subjects were included in the data analysis. One-year survival was $6.6 \%$, with a median survival of 56 days (95\% Cl: 46-67). The NLR had a discriminatory ability based on AUROC of 0.667 (95\% Cl: $0.536-0.798 ; p=0.044$ ), with the optimal cut-off point to differentiate survival was 3.7513 . The SII has a discriminatory ability based on AUROC of 0.766 (95\% Cl: $0.643-0.889 ; p=0.001)$, with the optimal cutoff point to distinguish survival was 954.4782 . SII had superiority in discriminatory ability $(p=0.0415)$.

\section{Conclusions}

The discriminatory ability based on AUROC of SIl was better than that of NLR in predicting one-year survival in patients with advanced HCC who did not undergo therapy.

\section{Introduction}

Hepatocellular carcinoma (HCC) is still an important disease burden in Indonesia. In addition to the high number of cases, one of the main problems with HCC is the low survival rate, mainly because patients only come seeking treatment when cancer has reached an advanced stage [1]. Treatment options that can be given at this stage are becoming increasingly limited, and it is not uncommon to only provide supportive therapy $[2,3]$. One of the main therapies, namely sorafenib, is inseparable from the potential for side effects, requires no small amount of money, and is not covered by national health insurance [4]. 
Therefore, it is necessary to have an accurate predictor marker to be able to identify which groups of patients may have better survival.

One of the predictors considered potential to be able to predict the prognosis of HCC patients is the parameters of the systemic immune/inflammatory response. Current research focuses on the combination/ ratio between several immune response parameters, especially neutrophils, lymphocytes, platelets, albumin, and $\mathrm{C}$ reactive protein [5-7]. Unfortunately, studies on these immune response parameters are still limited to the group of HCC patients who can still undergo curative therapy [8].

Inflammatory parameters assessed for potential in advanced HCC as predictors of current survival include neutrophil-lymphocyte ratio (NLR) and systemic immune-inflammation index (SII). Research results that have been published up to now generally still show inconsistencies and it has not been found which is the best parameter. In addition, there is no uniformity in the cut-off point value that can be used for each parameter $[9,10]$. Therefore, this study wanted to evaluate which of these immune response parameters could be used as an accurate predictor of survival in patients with advanced HCC. This knowledge will be important as reference material for consideration of palliative therapy in patients with advanced HCC.

\section{Methods}

\section{Patients and study design}

A retrospective database analysis was conducted on patients with advanced HCC (Barcelona Clinic Liver Cancer/BCLC C) who did not undergo therapy in two tertiary care hospitals (Cipto Mangunkusumo Hospital/RSCM and Dharmais Hospital/RSKD). The inclusion criteria for the study were patients aged more than 18 years, diagnosed with advanced HCC and did not undergo therapy, and had a complete peripheral blood examination as well as leukocyte differential count at the time of diagnosis. Patients with malignancies other than HCC, hematological disorders, human immunodeficiency virus (HIV), autoimmune disease, dengue fever, recipients of chemotherapy or long-term immunosuppressive therapy, or platelet transfusions within the last two weeks, were excluded from the study.

The research sample was taken using the consecutive sampling method from the hospital databases. The data collected included baseline characteristics (age, gender, comorbidity, infection status, etiology of HCC, performance status, liver cirrhosis status, Child-Pugh classification, alpha-fetoprotein/AFP level, number of nodules, nodule size, portal vein thrombus, and metastases), identification of prognostic factors (neutrophil count, lymphocyte count, NLR, platelet count, SII), and the data of one-year survival observed from the time of diagnosis. Survival data was acquired from the medical records, information from family relatives, or if both were not available, the patients were censored on the day of their last visit.

\section{Statistical Analysis}


The research data processing was carried out electronically using the SPSS device. Continuous data are expressed as mean value \pm standard deviation (SD) if the distribution is normal, otherwise as median value with minimal and maximal values. Categorical variables are expressed as absolute and relative frequencies.

The NLR and SII discrimination abilities as predictors of one-year survival were determined based on AUROC. AUROC comparison analysis between both parameters on the MedCalc device. The optimal cutoff point for NLR and SIl was determined by selecting the cut-off point with the best sensitivity and specificity based on the Youden index.

Survival analyses were calculated according to the Kaplan-Meier method in each group based on the cutoff point and compared by the log-rank test. A two-tailed $p$-value $<0,05$ was considered statistically significant. The cox regression statistical tests were performed to obtain crude HR values for NLR and SII. Infection status, comorbid status, and liver cirrhosis were considered as potential confounders. The confounding variables with a p-value $<0,25$ were analyzed with multivariate cox regression analysis. Those variables were considered as confounders only if they affected the crude HR of NLR and SII by more than $10 \%$.

\section{Ethical Clearance}

The ethical clearance was issued by the Ethics Committee of the Faculty of Medicine, Universitas Indonesia/ Cipto Mangunkusumo Hospital, with approval number ND-423/UN2.F1/ETIK/PPM.00.02/ 2021.

\section{Results}

\section{Patients' Baseline Characteristics}

From 319 patients who had an advanced HCC diagnosis according to the hospital databases, 196 patients were included in this study. The reason for the exclusion of the sample was generally due to treatment given to the patient, especially sorafenib and radiotherapy (Figure 1).

Research subjects had a median age of 54 years. The majority of research subjects were male (82.1\%). Comorbidity was found in $30.8 \%$ of study subjects. The most common comorbidities were hypertension, diabetes mellitus, and urinary tract stones, while infections were found in $14.8 \%$ of subjects, with the most infections being pneumonia and pulmonary tuberculosis. The majority of subjects had liver cirrhosis (81.6\%) and based on the calculation of the Child-Pugh score, it was found that $54.1 \%$ of the subjects had a Child-Pugh B score, with the main contributory components being ascites and hypoalbuminemia (Table 1 ). 
Table 1

Demographic and clinical characteristics of research subjects.

\begin{tabular}{|c|c|}
\hline Variable(s) & Study result(s) \\
\hline Age, median (min-max) & $53.5(19-82)$ \\
\hline \multicolumn{2}{|l|}{ Gender, n (\%) } \\
\hline - Male & $161(82.1 \%)$ \\
\hline • Female & $35(17.9 \%)$ \\
\hline \multicolumn{2}{|l|}{ Comorbidities, n (\%) } \\
\hline - None & $117(69.2 \%)$ \\
\hline - Present & $52(30.8 \%)$ \\
\hline \multicolumn{2}{|l|}{ Infection status, n (\%) } \\
\hline - None & $144(85.2 \%)$ \\
\hline • Present & $25(14.8 \%)$ \\
\hline \multicolumn{2}{|l|}{ Liver cirrhosis, n (\%) } \\
\hline • None & $36(18.4 \%)$ \\
\hline • Present & $160(81.6 \%)$ \\
\hline \multicolumn{2}{|l|}{ Ascites, n (\%) } \\
\hline • None & $79(46.7 \%)$ \\
\hline • Present, not massive & $77(45.6 \%)$ \\
\hline - Present, massive & $13(7.7 \%)$ \\
\hline \multicolumn{2}{|l|}{ Bilirubin, n (\%) } \\
\hline$\cdot<2 \mathrm{mg} / \mathrm{dL}$ & $127(65.1 \%)$ \\
\hline$\cdot 2-3 \mathrm{mg} / \mathrm{dL}$ & $41(21.0 \%)$ \\
\hline$\cdot>3 \mathrm{mg} / \mathrm{dL}$ & $27(13.8 \%)$ \\
\hline \multicolumn{2}{|l|}{ Albumin, n (\%) } \\
\hline$\cdot>3.5 \mathrm{~g} / \mathrm{dL}$ & $90(46.4 \%)$ \\
\hline - $2.8-3.5 \mathrm{~g} / \mathrm{dL}$ & $75(38.7 \%)$ \\
\hline$\cdot<2.8 \mathrm{~g} / \mathrm{dL}$ & $29(14.9 \%)$ \\
\hline PT/INR, n (\%) & \\
\hline
\end{tabular}




\begin{tabular}{|ll|}
\hline Variable(s) & Study result(s) \\
\hline$\cdot$ PT < 4 seconds and INR < 1.7 & $165(97.6 \%)$ \\
\hline One of PT 4-6 seconds or INR 1.7-2.3 & $3(1.8 \%)$ \\
\hline - One of PT > 6 seconds or INR > 2.3 & $1(0.6 \%)$ \\
\hline Encephalopathy, n (\%) & \\
\hline None & $160(95.2 \%)$ \\
\hline Mild-moderate (stage 1-2) & $5(3.0 \%)$ \\
\hline - Severe (stage 3-4) & $3(1.8 \%)$ \\
\hline Child-Pugh score classification, n (\%) & \\
\hline - Child-Pugh A & $90(45.9 \%)$ \\
- Child-Pugh B & $106(54.1 \%)$ \\
\hline
\end{tabular}

Hepatitis B was the main etiology (69.4\%) of HCC. A total of $70.8 \%$ of the subjects still had a good performance status, indicated by Eastern Cooperative Oncology Group (ECOG) grade 0-1. Transaminase levels of both alanine aminotransferase (ALT) and aspartate aminotransferase (AST) were generally found to be elevated, as were the levels of AFP. The tumor characteristics of the study subjects were more often found with multiple/diffuse nodules $(71.7 \%)$, large nodule size $(89.4 \%)$, portal vein thrombus (70.3\%), while metastases were found in $46.9 \%$ of subjects. Research subjects had a median NLR of 3.80 and SII of 1180.25 (Table 2). 
Table 2

Characteristics of research subjects related to tumor parameters

\section{Variable(s)}

Etiology of HCC, $n(\%)$

- Hepatitis B

- Hepatitis C

- Hepatitis B and C

- Non viral

Performance status, $\mathrm{n}(\%)$

- Grade 0-1

- Grade 2

Neutrophil levels, mean \pm SD

Lymphocyte levels, median (min-max)

Platelet levels, median (min-max)

AST levels, median (min-max)

ALT levels, median (min-max)

AFP levels $(\mathrm{ng} / \mathrm{mL})$, median (min-max)

Number of nodule(s), n (\%)

- Solitary

- Multiple/diffuse

Largest nodule size, $\mathrm{n}(\%)$

$\cdot \leq 5 \mathrm{~cm}$

$\cdot>5 \mathrm{~cm}$

Portal vein thrombus, $\mathrm{n}(\%)$

- None

- Present

Metastasis, n (\%)

- None

- Present

Neutrophil-lymphocyte ratio, median (min-max)
$19(10.6 \%)$

$161(89.4 \%)$

Study result(s)

$134(69.4 \%)$

$36(18.7 \%)$

$4(2.1 \%)$

$19(9.8 \%)$

$138(70.8 \%)$

$57(29.2 \%)$

$71.45 \pm 11.85$

$16.2(1.3-45.1)$

$254,500(35,000-773,000)$

$102.0(22-468)$

5.,5 (10-408)

$1,609.31(1.03-222,677.2)$

$54(28.3 \%)$

$137(71.7 \%)$

$58(29.7 \%)$

$137(70.3 \%)$

$104(53.1 \%)$

$92(46.9 \%)$

$3.80(1.00-41.86)$ 


\begin{tabular}{|ll|}
\hline Variable(s) & Study result(s) \\
\hline Systemic immune-inflammation index, median (min-max) & $1,180.25(126.36-13,310.32)$ \\
\hline One-year survival, n (\%) & $13(6.6 \%)$ \\
\hline - Alive & $183(93.4 \%)$ \\
• Died & \\
\hline Six-months survival, n (\%) & $32(16.3 \%)$ \\
• Alive & $164(83.7 \%)$ \\
\hline - Died & \\
\hline Three-months survival, n (\%) & $65(33.2 \%)$ \\
• Alive & $131(66.8 \%)$ \\
\hline D Died & \\
\hline
\end{tabular}

\section{Survival Analysis}

We managed to obtain exact survival data on 110 research subjects through medical record data or related hospital registers, phone contact with family members, while 86 other subjects who did not return to our hospital and could not be contacted were included in the sensor category based on the date of the last hospital visit.

Based on the Kaplan-Meier survival analysis, one-year survival was $6.6 \%$, six-month survival was $16.3 \%$, and three-month survival was $33.2 \%$. The median survival obtained was 56 days ( $95 \% \mathrm{Cl}: 46-67)$, with a range of 7-365 days (Figure 2). Causes of death could only be obtained from 46 medical records of study subjects, with the order of cause of death being tumor-related progression in 23 patients (50.0\%), gastrointestinal bleeding in 13 patients (28.3\%), and infection in 10 patients $(21.7 \%)$.

Based on one-year survival, the characteristics of the research subjects were further analyzed and compared between the two groups. Based on bivariate analysis, it was found that NLR and SII had a statistically significant median difference $(p=0.044$ and 0.001 , respectively, Table 3$)$. 
Table 3

Characteristics of research variables based on one-year survival.

\begin{tabular}{|c|c|c|c|}
\hline Variable(s) & Alive & Died & $\begin{array}{l}\mathrm{p}- \\
\text { value }\end{array}$ \\
\hline Neutrophil levels, mean \pm SD & $65.39 \pm 10.96$ & $71.88 \pm 11.82$ & 0.056 \\
\hline $\begin{array}{l}\text { Lymphocyte levels, median (min- } \\
\text { max) }\end{array}$ & $20.0(12.8-33.8)$ & $16.0(1.3-45.1)$ & 0.050 \\
\hline Platelet levels, median (min-max) & $\begin{array}{l}182,000(53,000- \\
375,000)\end{array}$ & $260,000(35,000-773,000)$ & 0.011 \\
\hline NLR, median (min-max) & $3.36(1.32-6.08)$ & $3.89(1.00-41.86)$ & 0.044 \\
\hline SII, median (min-max) & $433.45(126.36-668.30)$ & $\begin{array}{l}1,277.19(139.35- \\
13,310.32)\end{array}$ & 0.001 \\
\hline \multicolumn{4}{|l|}{ Comorbidities, n (\%) } \\
\hline - None & 5 & 112 & 0.077 \\
\hline - Present & 6 & 46 & \\
\hline \multicolumn{4}{|l|}{ Infection status, n (\%) } \\
\hline - None & 9 & 135 & 0.743 \\
\hline - Present & 2 & 23 & \\
\hline \multicolumn{4}{|l|}{ Liver cirrhosis, n (\%) } \\
\hline - None & 2 & 34 & \\
\hline - Present & 11 & 149 & 0.774 \\
\hline
\end{tabular}

The Discrimination Ability of NLR and SII as Predictors of One-Year Survival in Patients with Untreated Advanced HCC

The performance of NLR as a predictor of one-year survival in advanced HCC patients was analyzed using the ROC curve, where the AUROC result was 0.667 (95\% Cl: 0.536-0.798; $p=0.044$, Figure 3 ). The optimal cut-off point for NLR was determined with the help of the best Youden index evaluation, which was 3.7513 , with a sensitivity of 0.607 and a specificity of 0.769 . As additional data, we also tried to evaluate NLR as a predictor of six-month survival, with results AUROC $=0.577$ (95\% Cl: 0.454-0.699; $p=$ 0.171), and also as a predictor of three-month survival, with results AUROC 0.605 (95\% Cl: 0.515-0.694; p $=0.017)$.

Using the cut-off point of NLR $>3.7513$, one-year survival is $2.6 \%$ versus $12.2 \%$ at NLR less than the cutoff point. Similarly, the median survival was also found to be shorter at NLR $>3.7513$, which was 44.0 days (95\% Cl: 33.5-54.5) compared to 84.0 (95\% Cl: 55.6-112.4). Statistical calculation found a 
significant difference with the $p$-value of the log-rank test $=0.002$ (Figure 4). Based on cox regression analysis, the hazard ratio for NLR > the cut-off point is 1.505 (95\% Cl: $1.084-2.089, p=0.015)$.

The performance of SII as a predictor of one-year survival in advanced HCC patients was analyzed using the ROC curve, where the AUROC result was 0.766 (95\% Cl: 0.643-0.889; $p=0.001$, Figure 5). The optimal cut-off point for SII was determined with the help of the best Youden index evaluation, which was 954.4782 , with a sensitivity of 0.658 and a specificity of 0.846 . As additional data, we also tried to evaluate SII as a predictor of six-month survival, with results AUROC $=0.659(95 \% \mathrm{Cl}: 0.540-0.777 ; p=$ $0.004)$, and also as a predictor of three-month survival, with results AUROC $0.597(95 \% \mathrm{Cl}: 0.506-0.688 ; p$ $=0.027)$.

Using the SII cut-off point $>954.4782$, the one-year survival proportion was $1.6 \%$ compared to $14.9 \%$ in SII less than the cut-off point. Similarly, the median survival was also found to be shorter in SII >954.4782, which was 48.0 days (95\% Cl: $38.6-57.4)$ compared to 78.0 (95\% Cl: 51.7-104.3). Statistically, it was found that there was a significant difference with the p-value of the log-rank test $<0.001$ (Figure 6). Based on cox regression analysis, the hazard ratio for SII > the cut-off point is 1.824 (95\% Cl: 1.276-2.610; $p=0.001)$.

The discriminatory capabilities of RNL and IIS based on AUROC were compared with each other using the MedCalc tool. The results of the analysis showed that there was a statistically significant difference in discriminatory abilities with $p=0.0415$ (95\% Cl: 0.004-0.193, Figure 7).

\section{Analyses of Confounding Factors}

The confounding variables determined in this study were comorbid status, infection status, and liver cirrhosis status of the research subjects. The calculation of the hazard ratio analysis on the three variables is summarized in Table 4, where it was found that only comorbid status had a p-value $<0.25$. Cox regression multivariate analysis showed that there were no variables that proved to be confounders for the findings on NLR and SII (Table 5).

Table 4

Hazard ratio analysis on potential confounders for NLR and SII

\begin{tabular}{|lll|}
\hline Potential confounders for NLR and SII & HR (95\% Cl) & p-value \\
\hline Comorbidities & $0.698(0.495-0.985)$ & 0.041 \\
\hline Infection status & $0.963(0.619-1.499)$ & 0.868 \\
\hline Liver cirrhosis & $1.126(0.775-1.636)$ & 0.534 \\
\hline
\end{tabular}


Table 5

The adjusted hazard ratio for NLR and SII after the addition of potential confounders.

\begin{tabular}{|lllll|}
\hline $\begin{array}{l}\text { Potential } \\
\text { confounders }\end{array}$ & $\begin{array}{l}\text { Adjusted HR } \\
(\mathbf{9 5 \%} \mathrm{Cl})\end{array}$ & $\begin{array}{l}\text { p- } \\
\text { value }\end{array}$ & $\begin{array}{l}\text { Change of crude } \\
\text { HR }\end{array}$ & Conclusions \\
\hline NLR $\geq$ cut-off & $\begin{array}{l}1.505(1.084- \\
2.089)\end{array}$ & 0.015 & - & \\
\hline - Comorbidities & $\begin{array}{l}1.583(1.145- \\
2.187)\end{array}$ & 0.005 & $0.078(5.2 \%)$ & $\begin{array}{l}<10 \%, \text { not } \\
\text { confounder }\end{array}$ \\
\hline SIl $\geq$ cut-off & $\begin{array}{l}1.824(1.276- \\
2.610)\end{array}$ & 0.001 & - & \\
\hline - Comorbidities & $\begin{array}{l}1.932(1.375- \\
2.714)\end{array}$ & 0.001 & $0.108(5.9 \%)$ & $<10 \%$, not \\
& & & & \\
\hline
\end{tabular}

\section{Discussion}

More than half of HCC patients in Indonesia are diagnosed at an advanced or late stage, as shown by studies by Loho et al. and Jasirwan et al. [1, 3]. Patient characteristics are highly dependent on local epidemiology, where the Asia Pacific region is generally dominated by the age of diagnosis under 60 years and the etiology of hepatitis B, while in the European and American populations it is dominated by more advanced age and the etiology of hepatitis C [11-13]. The majority of patients at this stage can only undergo supportive therapy, as a result of poor liver function reserve, number of multiple/diffuse nodules, large nodule size, portal vein thrombus, and metastases $[11,13]$.

The survival found in this study was shorter than the results in other studies. This is understandable because our patients were only given supportive therapy, so survival would be much different from studies involving subjects receiving palliative therapy, such as sorafenib $[3,14]$. In other studies that have a similar setting to ours, the baseline characteristics of the subjects such as the etiology of HCC, liver function reserve, and tumor parameter profile will be differentiators of survival $[11,15]$.

NLR is the ratio of neutrophils and lymphocytes that play an important role in the inflammatory process and tumor carcinogenesis. High levels of neutrophils are known to promote tumor adhesion and metastasis through the secretion of various growth factors, especially vascular endothelial growth factor (VEGF) and various proteases, while lymphocytes are known to play an important role in defense against tumors where low lymphocytes reflect a low host immune response to malignancy [16].

Previous studies regarding NLR in advanced HCC, in both the treated and untreated groups, have been shown to predict post-treatment survival well for overall survival and progression-free survival. The cutoff point commonly used was 3.0-4.0. A recent meta-analysis by Liu et al. summarizes studies assessing NLR performance in patients treated with sorafenib [9]. Patients with lower NLR levels were shown to have a better response to sorafenib. This NLR-related result is also in agreement with the findings of the study by Aino et al. in advanced HCC patients with untreated extrahepatic metastases $[17,18]$. 
SIl is a combination parameter of neutrophils, lymphocytes, and platelets which is still relatively new to use. The rationalization for platelets to be included in the score index is the ability of platelets to protect circulating tumor cells, induction of epithelial-mesenchymal transition, and promotion of extravasation of tumor cells to metastasize. Initially, SIl was popularized by Hu et al. for the evaluation of survival of HCC patients undergoing surgical therapy, where an SIl cut-off point $>330$ was found indicating poor survival prediction and a higher relapse rate [16].

Other studies related to the application of SII in advanced HCC are still limited. In the sorafenib-treated group, Gardini et al. and Conroy et al. showed that the predictive ability of overall survival, progressionfree survival, and better sorafenib response if the value of SII was lower than the optimal cut-off $[19,20]$. Zhao et al. looked at HCC patients who were only given supportive care, and the study found SII to be an independent prognostic factor for survival [21]. Until now, there has not been an optimal SIl cut-off that can consistently predict survival. The study by Gardini et al. used an SIl cut-off point of 360 . The cut-off value in advanced HCC patients is predicted to be higher, as found by the study of Conroy et al., especially in our study population group where patients were not on therapy.

The prognostic significance based on AUROC is generally considered good if it has an AUROC of more than 0.7 . With the findings of our study, it can be concluded that NLR does not have a good predictive ability of survival, and vice versa for SII. Studies comparing the performance of NLR and SII in advanced HCC are very limited. There have been no studies in untreated HCC patients, but there are several studies in sorafenib-treated patients. The study by Gardini et al. and Conroy et al., both showed the superiority of SIl compared to NLR in predicting survival $[19,20]$.

The main difference between SII and RNL is the platelet parameters. The results in this study reemphasized the importance of platelets to be included in the predictor index of advanced HCC survival. Platelet integration is considered to represent more fully the inflammatory environment and carcinogenesis that occurs in untreated advanced HCC. High platelet levels, according to Pavlovic et al., are associated with larger tumor size and poor survival in a wide variety of cancers [22]. HCC is a unique type of tumor because it can provide 2 types of images. The first feature is a pattern of thrombocytosis, in which large HCC together with residual liver cells can produce thrombopoietin, which mobilizes platelets from the bone marrow. The next feature is a pattern of thrombocytopenia, as a result of hypersplenism, impaired hepatic thrombopoietin production, and autoantibodies, generally associated with smaller HCC, lower albumin, and baseline fibrosis/cirrhosis [22].

Our study has several limitations. First, this study used a retrospective study design that relies on medical record data, so it may not fully correspond to the real condition of the patient if it was not properly or completely documented in the medical record. Second, researchers have tried to obtain survival data from medical records and contact the patient's family contact number recorded in the system, but there was still some survival data that must be assumed from the patient's last visit history. Third, the NLR and SII data analyzed in this study are only one-time data, and it is interesting to know the dynamics of the parameters that occur and their implications for potential predictors of survival. 
In conclusion, the discriminatory ability based on AUROC of SIl was better than that of NLR in predicting one-year survival in patients with advanced HCC who did not undergo therapy. Related to this, we recommend that in the future, further research be conducted regarding the prediction model for advanced HCC survival by including SII as a component, and research on the role of SII as a predictor of therapeutic response in $\mathrm{HCC}$ patients given systemic therapy can also be developed.

\section{Declarations}

Acknowledgments: We would like to express our gratitude to our advisors, Chyntia Olivia Maurine Jasirwan, Pringgodigdo Nugroho, Kuntjoro Harimurti, and Iris Rengganis, who gave us constructive input throughout this project.

Compliance with Ethical Standards: Approval was obtained from the ethics committee of Universitas Indonesia. The procedures used in this study adhere to the tenets of the Declaration of Helsinki.

Funding Information: The authors did not receive support from any organization for the submitted work.

Conflict of Interests: The authors whose names are listed immediately below certify that they have NO affiliations with or involvement in any organization or entity with any financial interest (such as honoraria; educational grants; participation in speakers' bureaus; membership, employment, consultancies, stock ownership, or other equity interest; and expert testimony or patent-licensing arrangements), or nonfinancial interest (such as personal or professional relationships, affiliations, knowledge or beliefs) in the subject matter or materials discussed in this manuscript.

Author Contributions: All authors contributed to the study conception and design. Material preparation, data collection and analysis were performed by Lutfie, and Imelda Maria Loho. The first draft of the manuscript was written by Lutfie and all authors commented on previous versions of the manuscript. All authors read and approved the final manuscript.

\section{References}

1. Loho IM, Hasan I, Rinaldi C, et al (2016) Hepatocellular carcinoma in a tertiary referral hospital in Indonesia: Lack of improvement of one-year survival rates between 1998-1999 and 2013-2014. Asian Pacific J Cancer Prev 17:2165-70. https://doi.org/10.7314/APJCP.2016.17.4.2165

2. Bruix J, Reig M, Sherman M (2016) Evidence-Based Diagnosis, Staging, and Treatment of Patients with Hepatocellular Carcinoma. Gastroenterology 150:835-53. https://doi.org/10.1053/j.gastro.2015.12.041

3. Jasirwan C, Hasan I, Sulaiman A, et al (2020) Risk factors of mortality in the patients with hepatocellular carcinoma: A multicenter study in Indonesia. Curr Probl Cancer 44:100480

4. Kikuchi L, Chagas A, Alencar R, et al (2017) Adherence to BCLC recommendations for the treatment of hepatocellular carcinoma: impact on survival according to stage. Clinics 72:454-60. 
https://doi.org/10.6061/clinics/2017(08)01

5. Qi X, Li J, Deng H, et al (2016) Neutrophil-to-lymphocyte ratio for the prognostic assessment of hepatocellular carcinoma: A systematic review and meta-analysis of observational studies. Oncotarget 7:45283-301. https://doi.org/10.18632/oncotarget.9942

6. Li MX, Bi XY, Li ZY, et al (2015) Prognostic role of glasgow prognostic score in patients with hepatocellular Carcinoma: A systematic review and meta-analysis. Med (United States) 94:1-9. https://doi.org/10.1097/MD.0000000000002133

7. Yang R, Chang Q, Meng X, et al (2018) Prognostic value of Systemic immune-inflammation index in cancer: A meta-analysis. J Cancer 9:3295-302. https://doi.org/10.7150/jca.25691

8. Zheng J, Cai J, Li H, et al (2017) Neutrophil to Lymphocyte Ratio and Platelet to Lymphocyte Ratio as Prognostic Predictors for Hepatocellular Carcinoma Patients with Various Treatments: A MetaAnalysis and Systematic Review. Cell Physiol Biochem 44:967-81. https://doi.org/10.1159/000485396

9. Liu L, Gong Y, Zhang Q, et al (2020) Prognostic Roles of Blood Inflammatory Markers in Hepatocellular Carcinoma Patients Taking Sorafenib. A Systematic Review and Meta-Analysis. Front Oncol 9:1-10

10. Wang B, Huang Y, Lin T (2020) Prognostic impact of elevated pre-treatment systemic immuneinflammation index (SII) in hepatocellular carcinoma: A meta-analysis. Medicine (Baltimore) 99:1-7

11. Sinn DH, Cho JY, Gwak GY, et al (2015) Different survival of barcelona clinic liver cancer stage C hepatocellular carcinoma patients by the extent of portal vein invasion and the type of extrahepatic spread. PLoS One 10:e0124434. https://doi.org/10.1371/journal.pone.0124434

12. Giannini EG, Farinati F, Ciccarese F, et al (2015) Prognosis of untreated hepatocellular carcinoma. Hepatology 61:184-90. https://doi.org/10.1002/hep.27443

13. Khalaf N, Ying J, Mittal S, et al (2017) Natural History of Untreated Hepatocellular Carcinoma in a US Cohort and the Role of Cancer Surveillance. Clin Gastroenterol Hepatol 15:273-81. https://doi.org/10.1016/j.cgh.2016.07.033

14. Gomaa Al, Hashim MS, Waked I (2014) Comparing staging systems for predicting prognosis and survival in patients with hepatocellular carcinoma in Egypt. PLoS One 9:1-11. https://doi.org/10.1371/journal.pone.0090929

15. Cabibbo G, Enea M, Attanasio M, et al (2010) A meta-analysis of survival rates of untreated patients in randomized clinical trials of hepatocellular carcinoma. Hepatology 51:1274-83. https://doi.org/10.1002/hep.23485

16. Hu B, Yang XR, Xu Y, et al (2014) Systemic immune-inflammation index predicts prognosis of patients after curative resection for hepatocellular carcinoma. Clin Cancer Res 20:6212-6222. https://doi.org/10.1158/1078-0432.CCR-14-0442

17. Aino H, Sumie S, Niizeki T, et al (2014) Clinical characteristics and prognostic factors for advanced hepatocellular carcinoma with extrahepatic metastasis. Mol Clin Oncol 2:393-8. https://doi.org/10.3892/mco.2014.259 
18. Aino H, Sumie S, Niizeki T, et al (2016) The systemic inflammatory response as a prognostic factor for advanced hepatocellular carcinoma with extrahepatic metastasis. Mol Clin Oncol 5:83-8

19. Gardini AC, Scarpi E, Faloppi L, et al (2016) Immune inflammation indicators and implication for immune modulation strategies in advanced hepatocellular carcinoma patients receiving sorafenib. Oncotarget 7:67142-9

20. Conroy G, Salleron J, Belle A, et al (2017) The prognostic value of inflammation-based scores in advanced hepatocellular carcinoma patients prior to treatment with sorafenib. Oncotarget 8:9585364

21. Zhao L, Yang D, Ma X, et al (2019) The Prognostic Value of aspartate aminotransferase to lymphocyte ratio and systemic immune-inflammation index for Overall Survival of Hepatocellular Carcinoma Patients Treated with palliative Treatments. J Cancer 10:2299-311

22. Pavlovic N, Rani B, Gerwins P, Heindryckx F (2019) Platelets as Key Factors in Hepatocellular Carcinoma. Cancers (Basel) 11:1-17

\section{Figures}

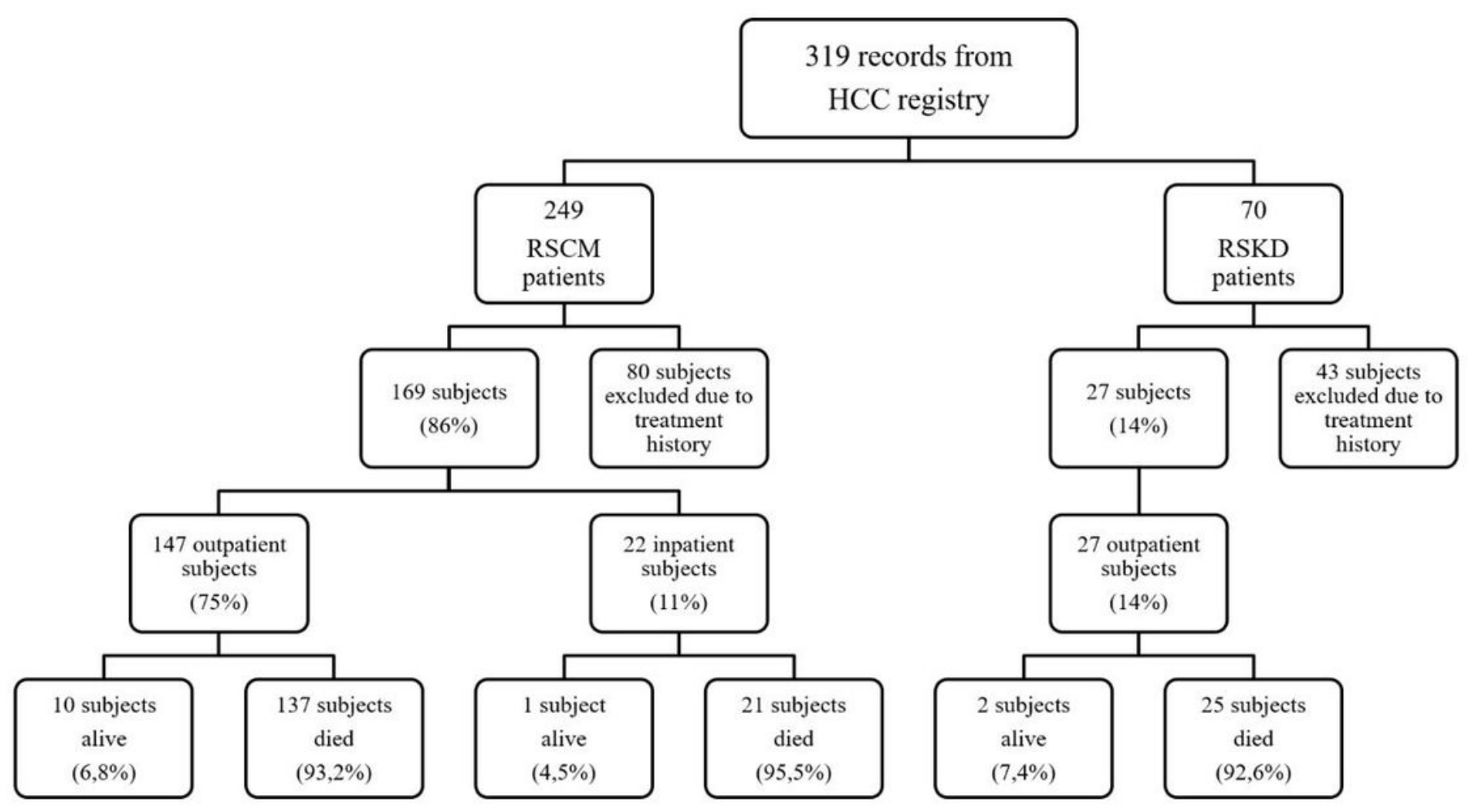

Figure 1

Flow Diagram Showing the Patient Selection. 


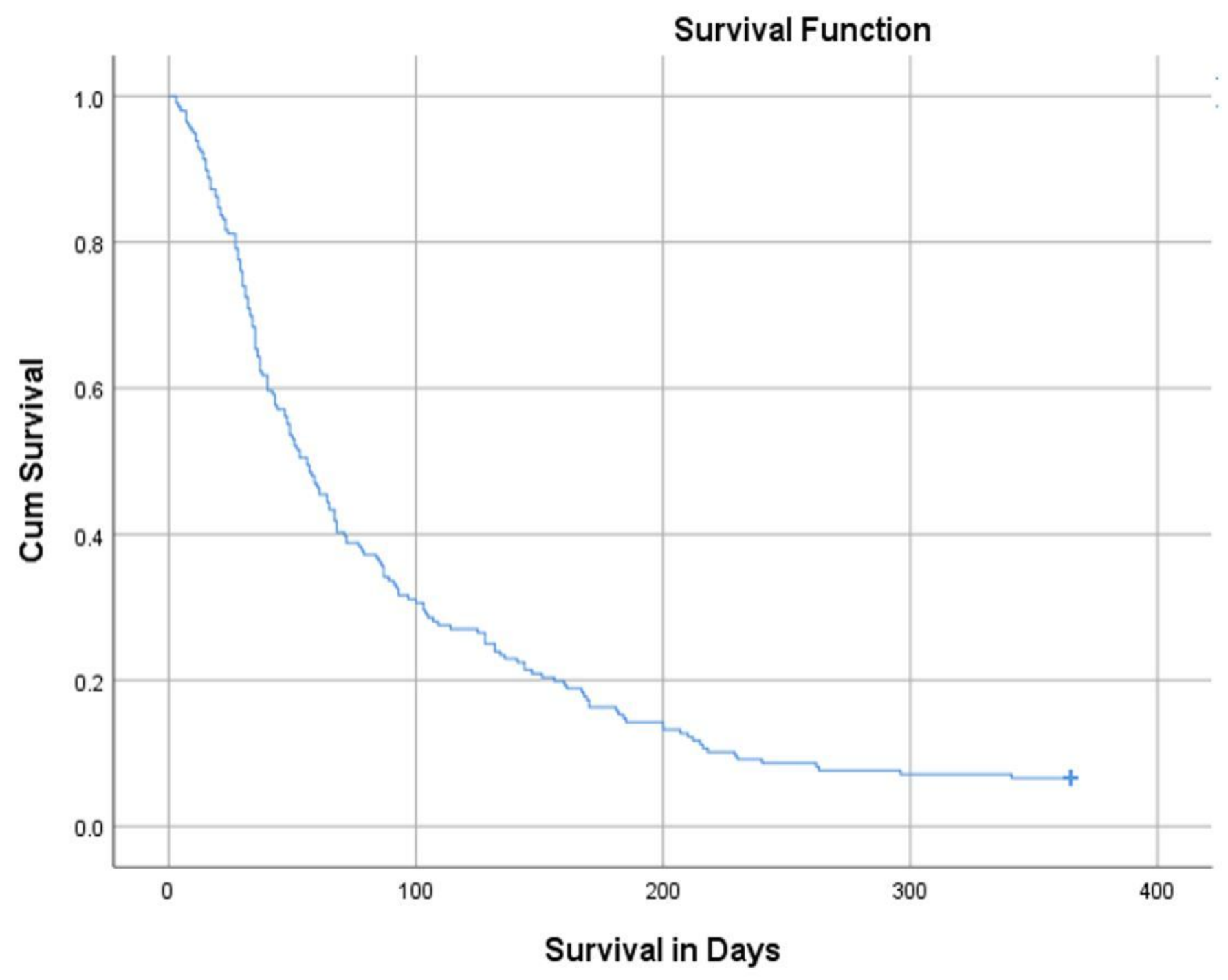

Figure 2

Kaplan-Meier curve showing cumulative survival in untreated advanced HCC patients. 


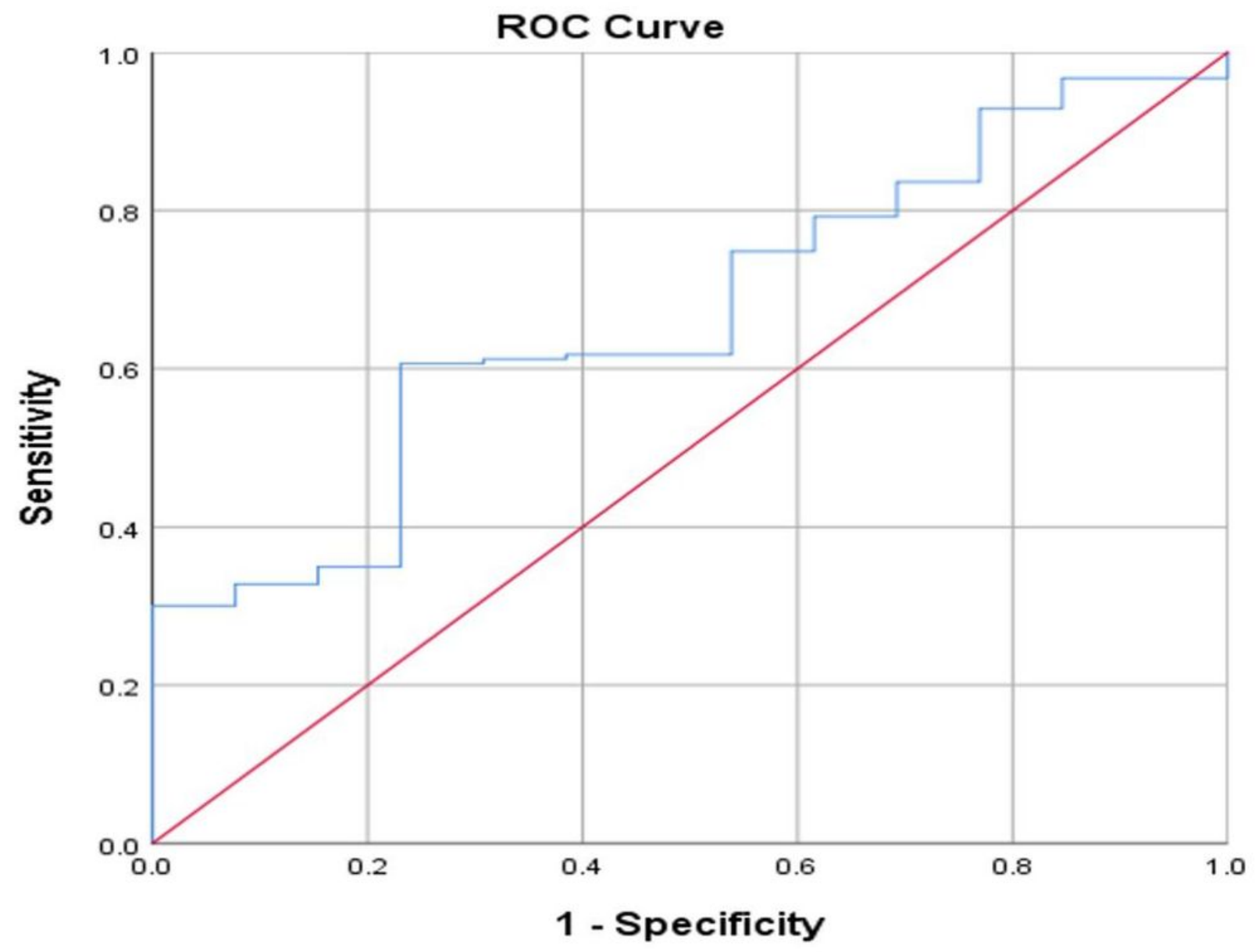

Figure 3

NLR ROC curve as a predictor of one-year survival in untreated advanced HCC patients. 


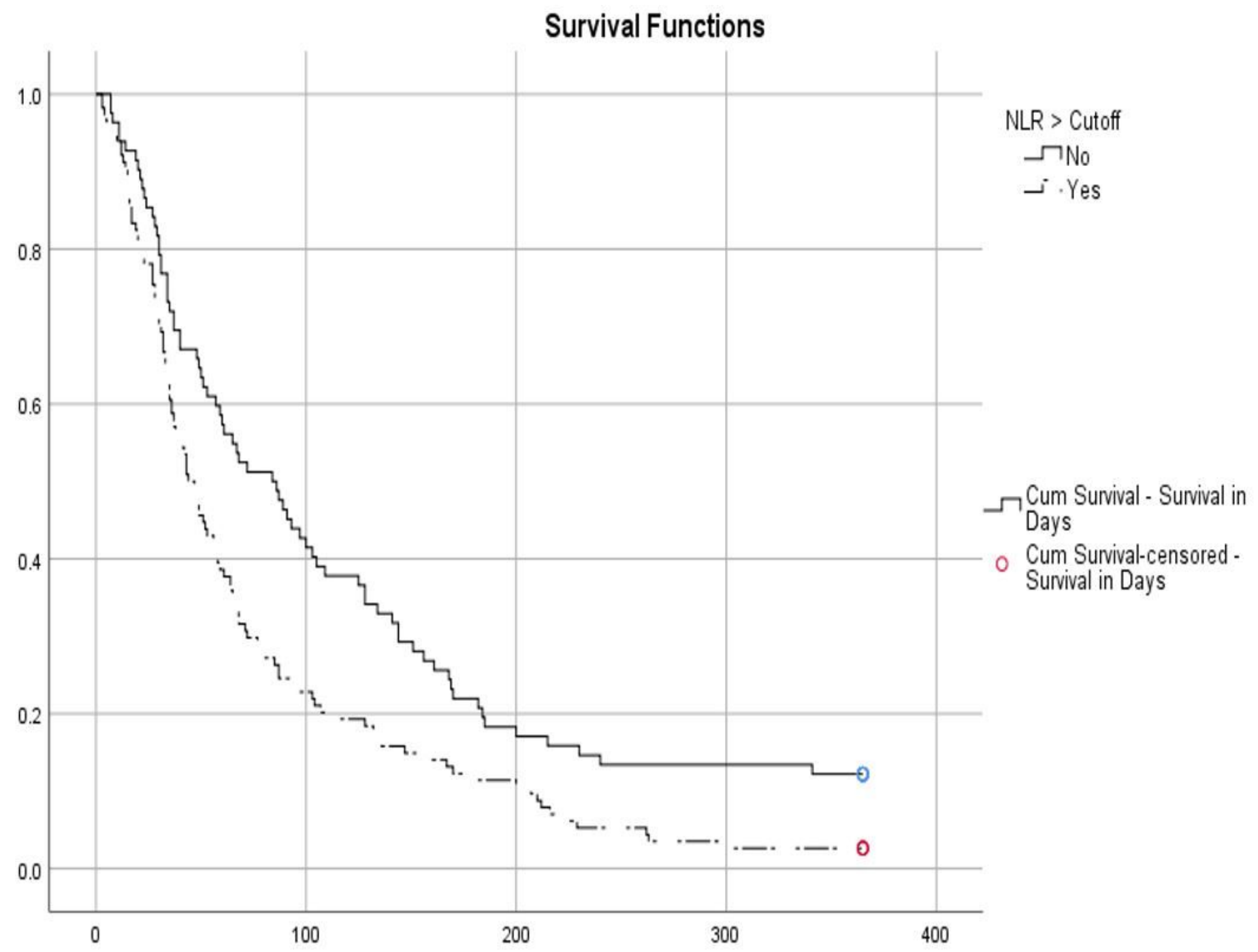

Figure 4

Kaplan-Meier curve showing the comparison of cumulative survival based on NLR in patients with untreated advanced HCC. 


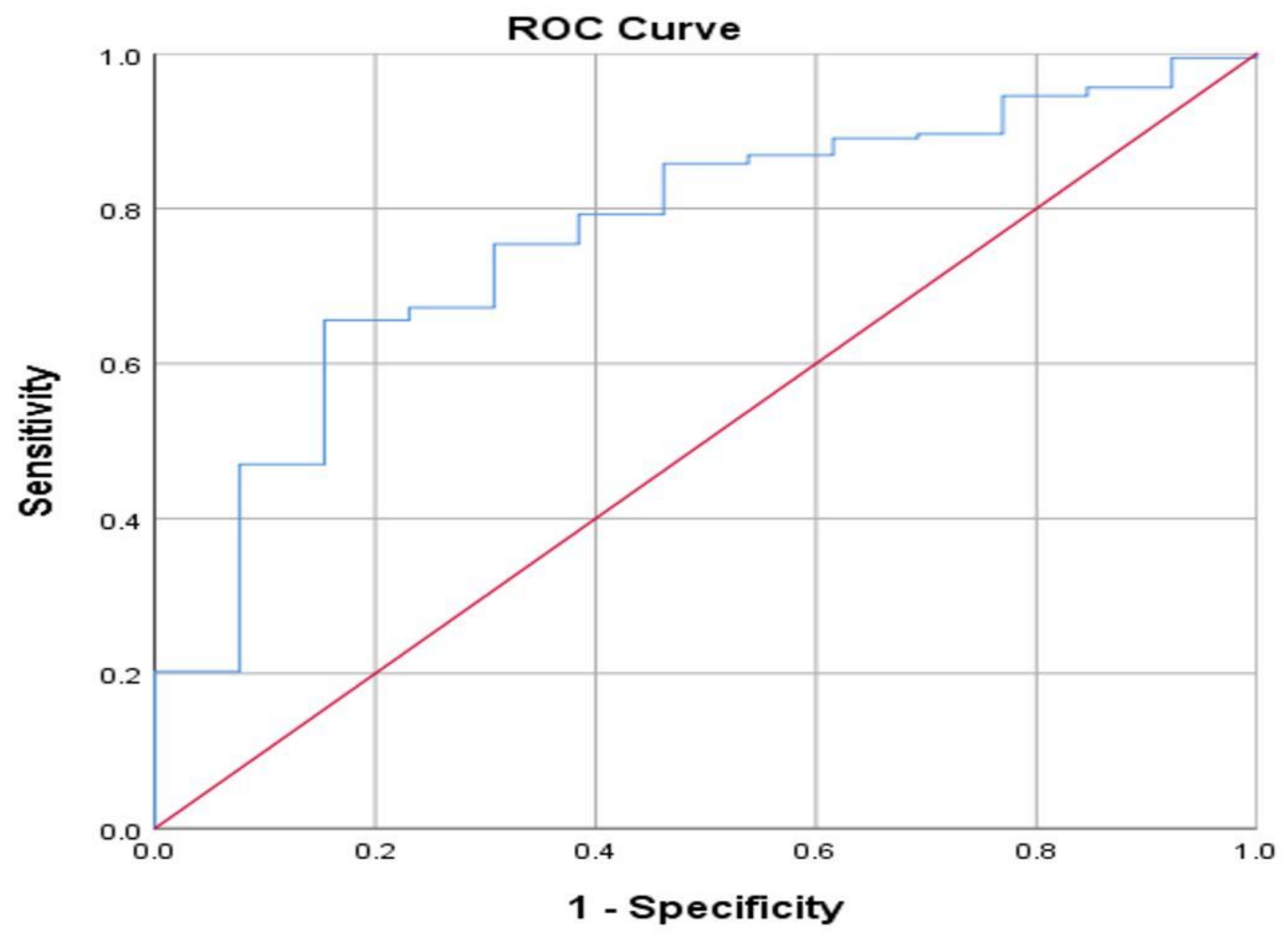

Figure 5

SII ROC curve as a predictor of one-year survival in untreated advanced HCC patients. 


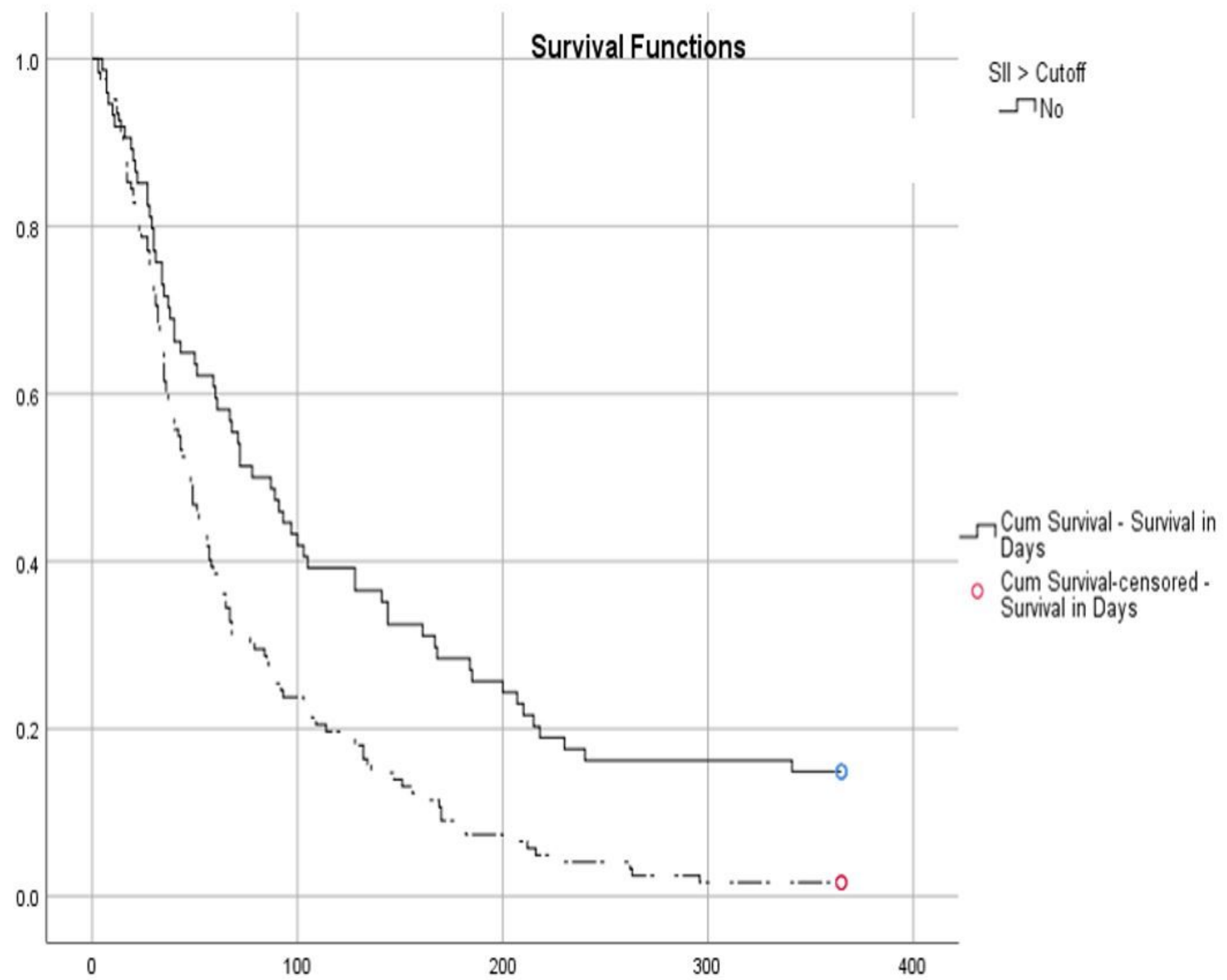

Figure 6

Kaplan-Meier curve showing the comparison of cumulative survival based on SII in patients with untreated advanced HCC. 


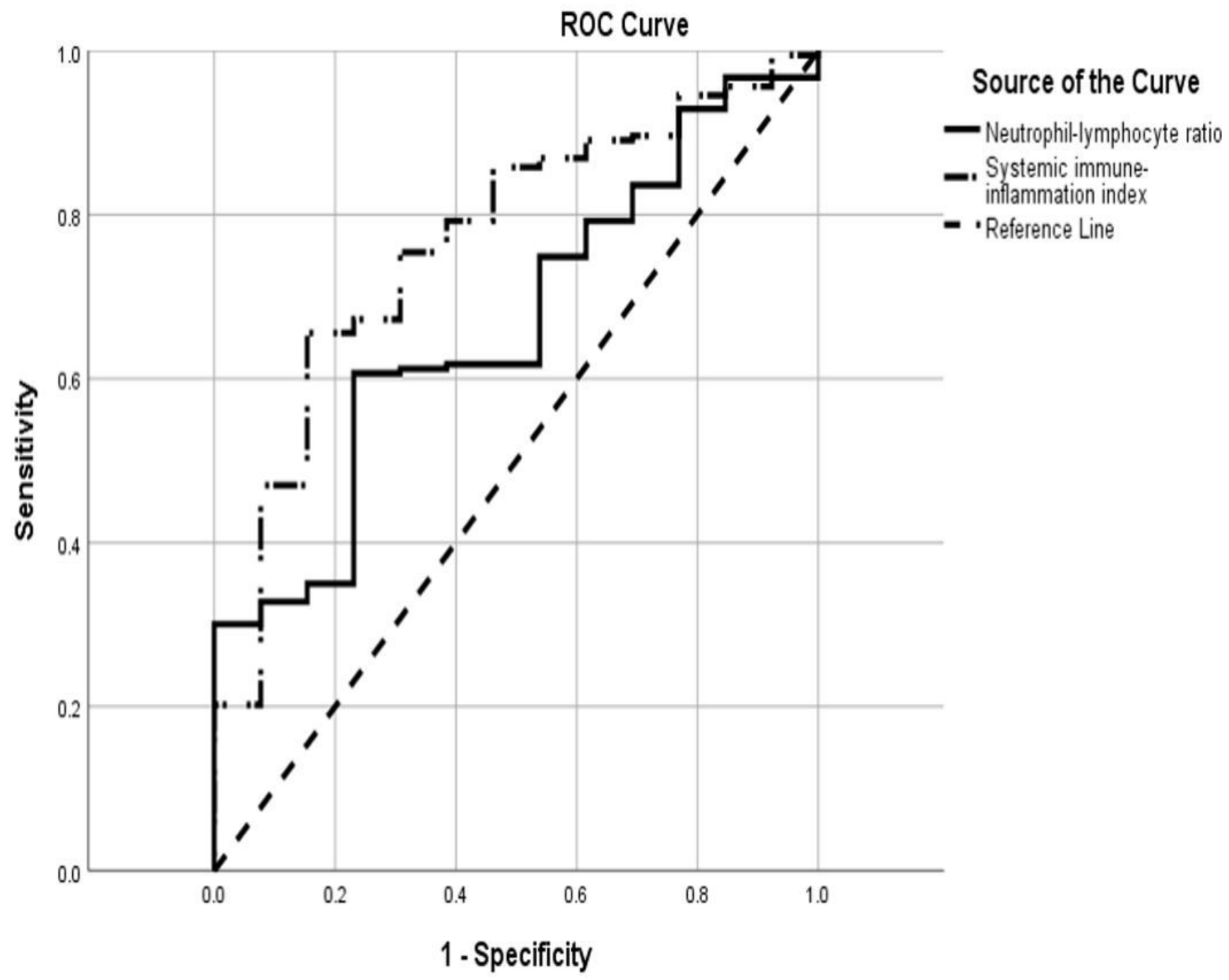

Figure 7

SIl compared to NLR ROC curve as a predictor of one-year survival in untreated advanced HCC patients. 